\title{
Unusual Presentation of Foreign Body Aspiration as Pleural Effusion in an Infant
}

\author{
T. Abiramalatha $\cdot$ Anuradha Bansal $\cdot$ S. Sudha $\cdot$ \\ Urmila Jhamb
}

Received: 5 August 2013 / Accepted: 9 December 2013 /Published online: 11 January 2014

(C) Dr. K C Chaudhuri Foundation 2014

To the Editor: Foreign body aspiration presenting as isolated pleural effusion without an underlying pneumonia is rare. So far only one case has been reported [1].

An 11-mo-old boy presented with a history of sudden onset of fast breathing and retractions since last $4 \mathrm{~h}$. He was hemodynamically stable with $95 \%$ saturation under hood oxygen. Auscultation revealed decreased air entry on the left side. Chest radiography showed pleural effusion with collapse lung on left side with ipsilateral mediastinal shift. Ultrasound chest showed left sided pleural fluid of $6 \mathrm{~mm}$ depth. Diagnostic pleural tap showed a transudative fluid with no pus cells or bacteria. Total blood WBC count was 18,600 with $78 \%$ polymorphs. The child had a fever spike of $101^{\circ} \mathrm{F} 2 \mathrm{~h}$ after admission. Hence, a possibility of community acquired pneumonia with synpneumonic effusion was kept and child was started on intravenous ceftriaxone.

After $48 \mathrm{~h}$ of treatment, the child's respiratory distress did not improve and air entry was persistently decreased on the left. Blood culture and pleural aspirate culture were sterile, no further fever spikes. Repeat chest radiography showed a partially expanded lung with only minimal blunting of costophrenic angle on the left and hyperinflated lung on the right. Hence, on clinical grounds, a diagnostic fibreoptic bronchoscopy was done which revealed a Bengal gram piece in the left intermediate bronchus. Urgent rigid bronchoscopy was done and two pieces of Bengal gram were removed from the left intermediate bronchus. Post procedure the child was treated with intravenous steroids and adrenaline nebulisation. The child showed dramatic improvement, respiratory distress settled. Repeat chest radiography $12 \mathrm{~h}$ later was normal. Blood counts normalised to a total count of 10,600 with $63 \%$ polymorphs and the child was discharged after $48 \mathrm{~h}$.

T. Abiramalatha $(\triangle) \cdot$ A. Bansal $\cdot$ S. Sudha $\cdot$ U. Jhamb Department of Pediatrics, Maulana Azad Medical College, New Delhi 110002, India

e-mail: abi_paeds@yahoo.com
There is a case report of a 60 -y-old man presenting with pleural effusion due to a candy wrapper aspiration [2]. But the effusion was secondary to pneumonia. Foreign body aspiration presenting as isolated pleural effusion is rare. So far only one case has been reported, a 3-y-old child with a vegetable foreign body presented with pleural effusion without pneumonia [1]. $750 \mathrm{~mL}$ of transudative pleural fluid was drained and the vegetable matter was removed by bronchoscopy.

The exact mechanism is not known. The possible mechanisms are either it may be a result of an inflammatory response to a foreign body in the bronchus or it is secondary to the atelectasis and the sudden more-negative intrathoracic pressure.

To conclude, in our case, poor response to conventional treatment, persistently decreased air entry and differential inflation even after the resolution of pleural effusion prompted us to investigate for a possible foreign body aspiration. This possibility should always be considered in any child presenting with respiratory symptoms not responding to conventional management.

Contributions TA and AB: Wrote the manuscript and did literature search. SS and UJ: Gave their valuable inputs. All were involved in management of the patient. UJ will act as guarantor for the paper.

Conflict of Interest None.

Role of Funding Source None.

\section{References}

1. Auerback ML. Pleural effusion due to unsuspected aspiration of vegetable matter in a 3-y-old boy. N Engl J Med. 1990;322:1238.

2. Antoun SA, Wehbe E. Pleural effusion from a candy wrapper. Cleve Clin J Med. 2009;76:639-40. 